\title{
Development and Evaluation of a Multimodal Touchpad for Advanced In-Vehicle Systems
}

\author{
Roman Vilimek ${ }^{1}$ and Alf Zimmer ${ }^{2}$ \\ ${ }^{1}$ Siemens AG, Corporate Technology, User Interface Design \\ Otto-Hahn-Ring 6, 81730 Munich, Germany \\ roman.vilimek. ext@siemens.com \\ ${ }^{2}$ University of Regensburg, Department of Experimental Psychology \\ 93053 Regensburg, Germany \\ alf.zimmer@psychologie.uni-regensburg.de
}

\begin{abstract}
Multimodal interaction can substantially improve human-computer interaction by employing multiple perceptual channels. We report on the development and evaluation of a touchpad with auditory, tactile and visual feedback for in-vehicle applications. In a simulator study, we assessed its suitability for interacting with a menu-based on-board system and investigated the effects of uni-, bi- and trimodal feedback on task and driving performance, workload and visual distraction in comparison to a conventional rotary push-button. In summary our results show that users clearly benefit from additional non-visual feedback while driving. When using the touchpad with multimodal feedback, our subjects also reached a higher level of performance compared to the rotary push-button.
\end{abstract}

Keywords: Multimodal, haptics, tactile feedback, auditory feedback, driving.

\section{Motivation}

In recent years, the automotive industry had to face the challenge of integrating a huge number of additional functions for driver information, communication and navigation in modern cars. To avoid an excessive amount of controls and displays, a growing number of car manufacturers use a computer-like menu-based system with a display in the center console and a central control for manual input. This central control is in most cases a form of rotary push-button positioned between the front seats. As an alternative, we propose a touchpad-based central control with multimodal feedback to overcome some inherent drawbacks of the rotary push-button concept.

Using a touchpad to control the cursor movements offers several advantages. Menu items or interaction elements for setting functional parameters are typically not displayed in a circular layout. When using a rotary push-button, the user is required to convert the circular input movements to the intended linear movements of the cursor. This can easily get confusing when vertical arrangements of interaction elements like menus and horizontal arrangements typically used for sliders are intermixed. Furthermore, the rotary push-button does not allow for two-dimensional movements on the screen. Taken together, the rotary push-button limits the systematic application of 
natural mappings [1], one of the basic requirements for intuitive man-machine interaction. With a touchpad, cursor movement is simply controlled linearly in two dimensions by moving the finger in the desired horizontal or vertical direction.

However, a conventional touchpad is most likely not suitable for in-vehicle use as actions are only visible on the graphical user interface. To minimize visual distraction we built a touchpad with additional auditory and / or haptic feedback. Any event shown visually is also displayed redundantly in a non-visual modality. Auditory feedback is given by playing short auditory icons, i.e. natural sounds which are mapped to system events by analogy (e.g., click-sounds; for details see [2]). Appropriate freely programmable actuators are used to generate vibrotactile feedback in form of vibration patterns underneath the touchpad. If, for instance, the user moves the selection cursor from one menu element to the next, he hears a clicking sound and feels a short tactile impulse at his fingertip. Thus, the visually displayed selection of a new element becomes palpable and audible. When a menu item is activated, another sound or vibration pattern is played. Characteristics of the prototype are described below in more detail.

\section{Research Goals}

The goals of our study were twofold: 1) On a theoretical level we explored the effects of multimodal feedback, especially the relative contributions of auditory and haptic feedback in direct comparison; 2) On a practical level we strove for a more ergonomic solution to the problem of manual input for in-vehicle secondary tasks while driving, by providing the users with a touchpad instead of a rotary push-button.

According to Wickens' multiple resource model [3], an additional auditory display improves time-sharing performance in a multiple-task setting with high visual load. This prediction has been confirmed in various contexts (e.g. [4, 5]). Using a touchpad with additional auditory, tactile or auditory plus tactile output should thus allow the driver to perceive more driving-relevant information while interacting with the onboard system compared to a touchpad with visual feedback only. Furthermore it has been shown that ballistic targeting tasks can be accomplished faster with additional feedback $[6,7]$. However, several aspects need closer analysis.

Whereas there is substantial evidence of the advantageous effects of multimodal feedback in general, there is no universal multimodal theory that allows the deduction of success probabilities for a novel interface in a concrete task environment. The multiple resource model provides a general framework for theoretical considerations, but in practice too many factors influence the interaction between users and a particular novel interface solution. Thus, the anticipated positive effects of multiple feedback modalities for a touchpad-based central control for on-board systems need experimental confirmation.

Based upon previous research it seems very likely that auditory feedback is an appropriate and accepted way of providing additional information to cursor movements. However, it is unclear to what extent users will benefit from vibrotactile information. Tactile displays are not new, but have mainly been used for highly specialized applications such as displays for blind people. However, as the cutaneous sense is still under-utilized in human-computer interaction [8] there is not sufficient knowledge on 
how to design vibrotactile patterns as a means of informing the user on the success of intended interaction events. Systematic research on the use of structured, abstract vibrotactile patterns to communicate messages non-visually (so-called Tactons or tactile icons) has just started in the recent past $[9,10]$. The present study examines whether Tactons are as effective as auditory messages in providing non-visual action feedback.

As an external baseline we included a rotary push-button in our study. We used a standard conventional rotary push-button switch, i.e. menu items are selected by turning the switch, and the selection is confirmed or activated by pressing it. There was no advanced auditory or haptic feedback for this input device, but in addition to the visual feedback on the graphical user interface (GUI) the mechanical properties of the switch enabled the users to infer important information non-visually. For instance, when turning the switch they feel the detent and hear a clicking sound when it snaps into position. Our subjects had to accomplish several experimental tasks using either this rotary push-button or the touchpad with unimodal (visual only), bimodal (visual+tactile or visual+auditory) and trimodal (visual+tactile+auditory) feedback. This experimental setup allows for direct comparisons between the different input devices and the effects of multimodal feedback.

\section{The Multimodal Touchpad}

The multimodal touchpad was evaluated in a cockpit mock-up. It was placed between the two seats and replaced the rotary push-button as central input device. In our experimental tasks, subjects had to accomplish several tasks which are typical for on-board systems. If, for instance, they wanted to use entertainment functions, they first had to select the menu entry "entertainment" in the main menu by moving their finger up or down in order to place the cursor onto the corresponding element. A short tap on the touchpad then activated the selection. Moving the cursor and clicking on the touchpad are similar to the actions performed with an ordinary laptop touchpad. However, the cursor did not move freely like a mouse cursor. It was used as a selection cursor, visible on the GUI as a highlighted frame around a menu item or any other selectable control element and restricted to move on these elements. In addition to the visual indication of movement, tactile feedback for the touchpad was realized by using unbalanced electric motor vibrators and a tactile transducer (bass shaker) mounted on a plate below the touchpad and a wrist rest. The wrist rest supported the users arm while he or she performed input operations. Different forms of tactile impulses were designed for output at the user's index finger and wrist. Figure 1 shows the components of the touchpad prototype and its integration into the cockpit mock-up which was used for the driving simulation. It also shows the TFT display next to the steering wheel for visual output and the loudspeaker behind the displays for auditory feedback.

The tactile transducer was a Fischer Amps shaker (type "Bass Pump III") specialized for low frequency vibrations, which was amplified with an additional low frequency amplifier (AURA Interactor) and connected to one of the soundcards of a PC. It was positioned right under the touchpad. The unbalanced electric motor vibrators under the wrist rest were those of a Logitech® ${ }^{\circledR} \operatorname{umblepad}^{\mathrm{TM}} 2$, which was connected 


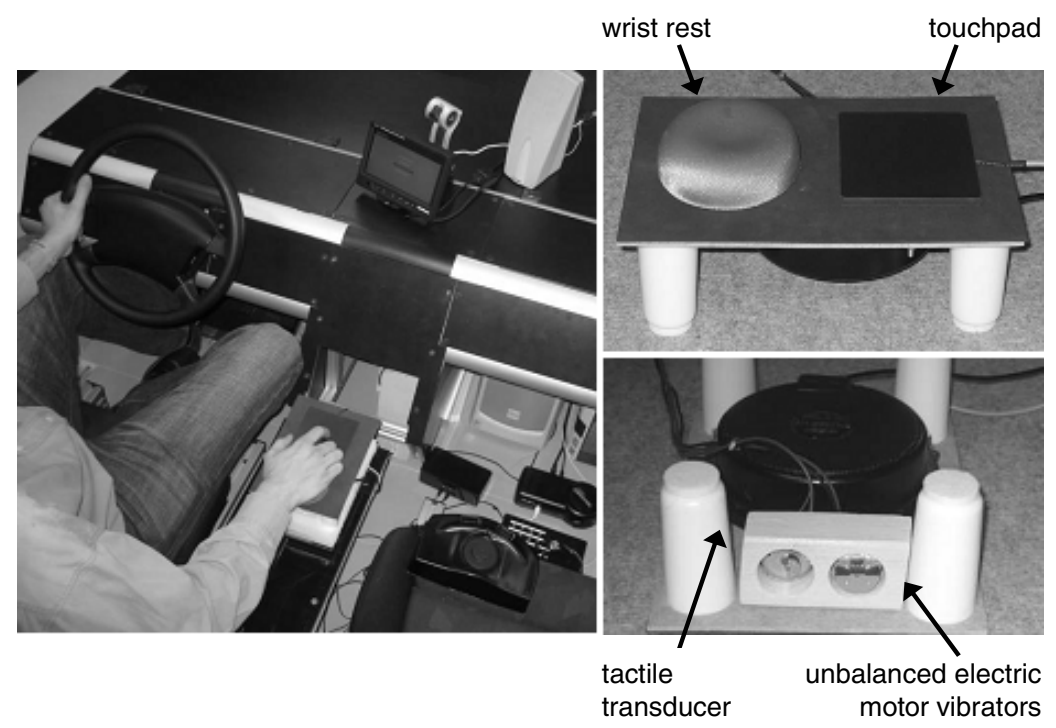

Fig. 1. Left side: Cockpit mock-up. Right side: Constituent parts of the multimodal touchpad.

to the PC via USB. The motors were generally used for slower but smoother feedback at the wrist whereas the tactile transducer generated single, short impulses at the fingertip. The tactile feedback events were designed either with the software Immersion Studio ${ }^{\circledR}$ by Immersion, Corp. (for the motors) or consisted of simple low frequency sine waves generated with the help of the sampling software Cool Edit 2000 (Syntrillium Software Corporation).

Any feedback event shown visually was also implemented in the non-visual modalities. In our experiment, several different types of events had to be designed. Most important are the selection and the confirmation/activation of an item, which are described here in detail. A movement of the selection cursor was signaled by a $20 \mathrm{~ms}$ $50 \mathrm{~Hz}$ sinus impulse issued by the tactile transducer. The auditory feedback of this event was a metallic clicking sound $(15 \mathrm{~ms})$. The confirmation/activation of a selected element by tapping on the touchpad was represented auditorily with a deep knocking sound, like knocking on a table $(60 \mathrm{~ms})$. Tactile feedback consisted of a $20 \mathrm{~ms} 30 \mathrm{~Hz}$ sinus wave and additional activity of the motor vibrators for $150 \mathrm{~ms}$. As the index finger had to be lifted off the touchpad during tapping, the tactile feedback was primarily perceived as a vibration of the wrist rest.

Considerable care was taken to ensure that tactile events were well perceivable but nevertheless did not produce audible artifacts. The frequency range was restricted to very low frequencies. The plate with the touchpad and the tactile actuators was placed on a special pad which absorbed the structure-borne sound. Thus, the effects of tactile feedback were only marginally contaminated with effects of auditory perception. 


\section{Methods}

\subsection{Participants}

Twenty participants (twelve female, eight male) took part in the present study. They were monetarily compensated or received a small gift for their participation. Their ages ranged from 22 to 36 years. All had held a valid driving license for at least two years and drove a car regularly. This was essential because the simulated driving task had to be dealt with and prioritized in the same way as real driving.

\subsection{Apparatus and Driving Simulation}

The study took place in the Corporate Technology usability lab at Siemens AG. The subject's workplace during the experiment was the driver's seat of a fixed-base cockpit mock-up. Depending on the experimental condition, the touchpad or the rotary push-button was positioned between the seats to the right of the driver. The experiment was run on a Fujitsu Siemens PC with $3.4 \mathrm{GHz}$ CPU equipped with two identical ESS Technologies ESS1969 Soundcards (one auditory, one for vibrotactile feedback). The experimental conditions were controlled by a Microsoft ${ }^{\circledR}$ Visual Basic ${ }^{\circledR}$ program that generated the interface, processed the user inputs, controlled the multimodal feedback and logged all user actions. The central display ( 7 " TFT running at a resolution of $800 \times 480$ ) of the simulated on-board system was placed on the dashboard to the right of the steering wheel. Figure 2 shows the setup with either the touchpad or the rotary push-button. Auditory feedback was issued by a loudspeaker behind the display.
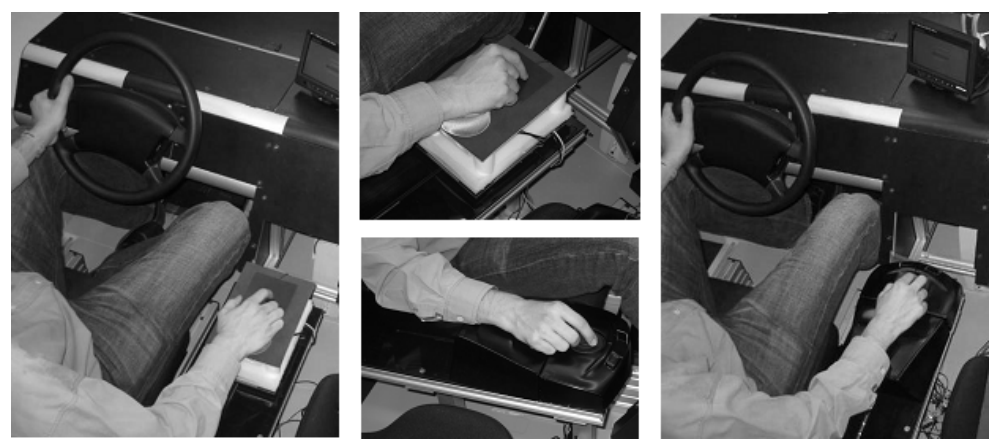

Fig. 2. Experimental setup with multimodal touchpad (left) and rotary push-button (right)

For the baseline comparison, a rotary push-button switch was vertically mounted in a case (ddm hopt+schuler, type $427 ; 30$ positions, $360^{\circ}$ endless, operating torque: $1.5 \mathrm{Ncm}$, actuation force: $6 \mathrm{~N}$, detent angle: $12.5^{\circ}$ ). The shaft was covered with a round plastic cap (approximately $4 \mathrm{~cm}$ in outside diameter).

To assess visual distraction a digital video camera (Philips SPC600NC) connected to a separate Fujitsu Siemens PC with 2.8 GHz CPU recorded the subjects' face (30 
frames/second; resolution: $640 \times 480$ ). After each experimental session, the video was analyzed manually in slow motion using a customized video tool.

The driving simulation used in this experiment was the Lane Change Task (LCT) [11]. The driving task consists of lane keeping and lane changing maneuvers in response to traffic signs at the side of the road. By comparing the driving performance with a normative model, the LCT allows the measurement of distraction caused by invehicle systems. It delivers a deviation score which covers important aspects of driving like the quality of maneuvers and the perception of driving-relevant information.

\subsection{Tasks}

The experimental tasks represented typical aspects of input operations with in-vehicle systems. The participants had to adjust numerical values for temperature or volume using horizontal or vertical sliders, select characters from a 2 dimensional array to enter a navigation destination, select items from a list and browse through different menus. In half of the experimental trials they had to drive concurrently. They were instructed to prioritize driving and treat it strictly as the primary task.

\subsection{Design}

This study was a $2 \times 5$ factorial, within-subject design. The independent variables were task situation and type of HMI. Task completion time, subjective mental workload (RTLX, a modified version of the NASA TLX [12]), driving performance (LCT) and display viewing times were the dependent variables.

All subjects interacted with the on-board system either in a single-task static test situation involving only the input tasks or in a dual-task situation with concurrent driving simulation (=independent variable: task situation). Furthermore, all subjects used all different HMI variants available in this study, as defined by the type of interaction device (touchpad or rotary push-button) and the type of feedback given when using the touchpad. Visual feedback was always present. Thus, the second independent variable, HMI, had five levels:

- TPv: Unimodal touchpad with only visual output without additional feedback

- TPva: Bimodal touchpad with visual and auditory feedback

- TPvt: Bimodal touchpad with visual and tactile feedback

- TPvta: Trimodal touchpad with visual, tactile and auditory feedback

- RPB: Rotary push-button switch

The subjects first completed all experimental tasks in five blocks, defined by the type of HMI used. A Latin square was used to counterbalance the block sequences with a new random sequence after any group of five subjects. In each block the experimental tasks were performed first without any driving task and after that with concurrent simulated driving. Additionally, all participants had to complete one LCT run without simultaneously using the on-board system to deliver a baseline measure of their driving performance. Subjects performed this task either at the beginning, in the middle or at the end of the experiment. 


\subsection{Procedure}

Before engaging in the experiment, the participants were pre-screened to ensure that they met the qualification criteria. The experimenter explained the experimental tasks and the driving task. The participants received a short driving training session. They also completed several practice runs with the on-board system.

Each experimental block started with a short practice run on the current HMI. After finishing all tasks with and without driving, the participants filled out the workload questionnaire at the end of each block. Finally, after finishing all experimental blocks and the baseline LCT, the participants were encouraged to report their opinions and preferences regarding the HMI variants.

\section{Hypotheses}

On the one hand, the better the natural mapping between cursor movements and GUI responses, the greater the likelihood that the touchpad will lead to more intuitive and thus faster interaction. On the other hand, this advantage may be substantially reduced by the increased visual load if the touchpad does not provide additional non-visual feedback. However, when the subject focuses all visual and cognitive resources on the single-task situation without driving, only marginal differences are expected between the rotary push-button and the touchpad, independently of additional feedback. But as soon as a primary visual task, the LCT, is present, the multiple resource model [3] implies a stronger decrease in performance for the secondary task if only visual presentation is available. Thus, the touchpad variants with bi- or trimodal feedback should have a general advantage over the unimodal visual-only touchpad. Furthermore it is very likely that the rotary push-button will also lead to better time-sharing than the unimodal touchpad because of its inherent mechanical feel and audible feedback. Considering basic research results [13], it also seems likely that any positive effects will be more pronounced with trimodal than with bimodal feedback.

\section{Results and Discussion}

Data collected in the present experiment were analyzed using inferential statistics (ANOVA). The alpha level for significance was chosen to be .05.

Regarding task completion times, no major differences were found between the different HMIs (TPv, TPva, TPvt, TPvta, and RPB) in the single-task situation. The values ranged between 1.97 and $2.07 \mathrm{~s}$. But when the subjects had to drive concurrently in the dual-task situation, a different picture emerged (see Fig. 3). Of course it took more time to fulfill the tasks regardless of the HMI used (significant main effect for task situation: $F(1,19)=34.86, p<.001)$. More interesting are the differences between the HMI variants while driving. If the subjects had only visual feedback when interacting with the touchpad they needed $3.41 \mathrm{~s}$. This is considerably longer than the mean task completion times for the bi- or trimodal touchpads, TPva: $2.82 \mathrm{~s}$, TPvt: $2.86 \mathrm{~s}$, TPvta: $2.96 \mathrm{~s}$. Completing a task with the rotary push-button took the participants almost as long as with the unimodal touchpad (3.39 s). 


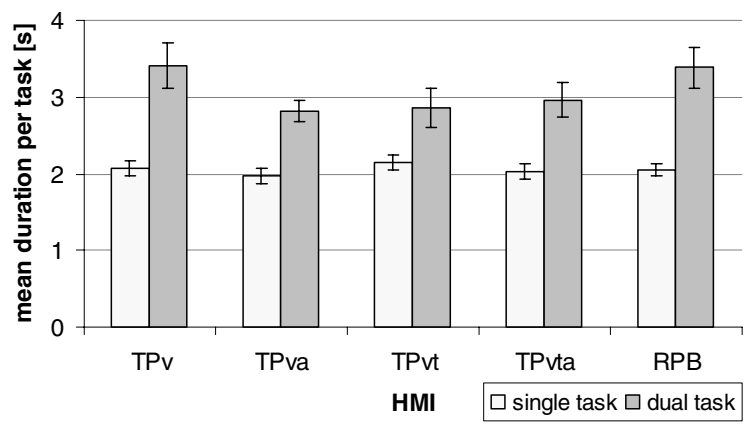

Fig. 3. Task completion times for each HMI variant in single-task (static test) and dual-task (with simulated driving) situations. Error bars indicate standard error of the mean.

A significant interaction between HMI and task situation $(F(4,76)=2.84, p=.03)$ confirms the hypothesis that the differences between the HMI variants show up only if there is a resource conflict in the dual-task situation. If additional feedback is available for the touchpad, task completion times are clearly shorter. Interestingly, there is no indication that trimodal feedback has a more pronounced effect than bimodal. Furthermore the relatively long task completion times of the baseline RPB are worth mentioning. Using the RPB took our subjects almost as long as using the unimodal touchpad TPv. Based upon these data, even a conventional touchpad with only visual feedback seems to be a reasonable alternative.

An analysis of the LCT data reveals that the subjects successfully prioritized the primary task: safe driving. The deviation from the normative model (optimal lane keeping and changing) was very similar in the baseline run without any additional task and when subjects were driving under dual-task conditions, i.e. the quotient of the deviation with/without secondary task is near 1. For each HMI, the average values are: TPv: 1.00, TPva: 1.00, TPvt: 1.03, TPvta: 1.05, RPB: 1.04. A one-factor repeated measures ANOVA did not indicate significant differences $(F(4,76)=1.7, p=.15)$.

To assess visual distraction, we analyzed the duration of gazes at the display during driving. The longest mean duration of a single gaze at the display (see Fig. 4, A) was found when subjects were interacting with the unimodal touchpad (TPv: 0.79 s), whereas the auditory touchpad produced the shortest control gazes (TPva: $0.66 \mathrm{~s}$ ). Accordingly, the remaining touchpad variants and the RPB fall within this range (TPvt: 0.73 s, TPvta: $0.70 \mathrm{~s}, \mathrm{RPB}: 0.67 \mathrm{~s}$ ). A one-factor ANOVA evaluated this effect of HMI and confirmed it as significant $(F(4,76)=2.46, p=.05)$. However, the differences between the HMI variants are rather small, so there may be doubt as to their practical impact. Taking account of the total time the subjects spent looking at the display (see Fig. 4, B) helps to evaluate the consequences of longer input operations.

Major differences between the HMI variants are obvious at first sight. They proved to be statistically meaningful $(F(4,76)=11.80, p<.001)$. These data provide evidence that the unimodal touchpad (TPv: $19.15 \mathrm{~s}$ ) leads to noticeably prolonged visual distraction compared to the baseline RPB (16.75 s), which limits its usefulness in practice. They also suggest a clear advantage of the bi- and trimodal touchpads over the baseline RPB (TPva: 11.84 s, TPvt: 14.20 s, TPvta: 13.35 s). 
(A)

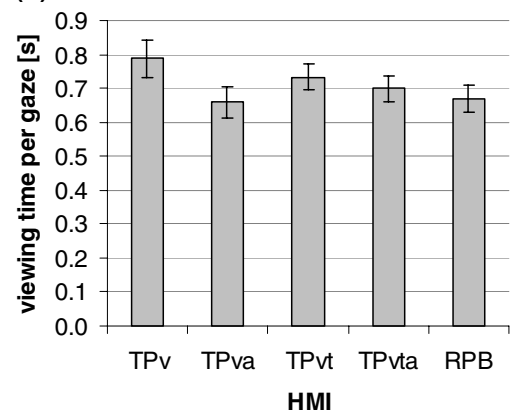

(B)

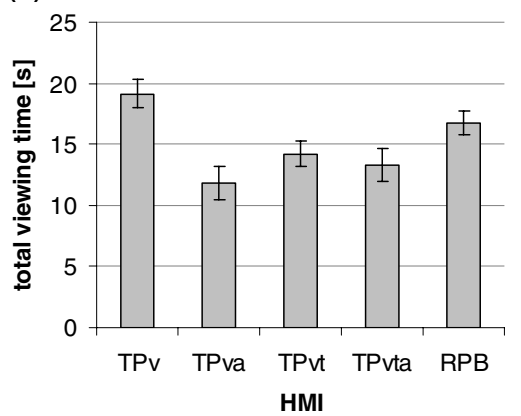

Fig. 4. A) Average duration of control gazes at the display and B) total viewing time accumulated over all experimental tasks for each HMI variant (with standard errors)

The subjective workload ratings deliver a similar pattern of results. The overall score of the RTLX ranges from 0 to 100, with higher values indicating a higher workload. Subjects reported the highest workload when using the unimodal TPv, 35.6, and the lowest when interacting with the auditory bimodal touchpad TPva, 23.9. An average value of 28.0 was found for the bimodal tactile touchpad TPvt, 24.9 for the trimodal touchpad TPvta and 29.8 for the rotary push-button RPB. A one-factor ANOVA showed significant differences between these HMI variants $(F(4,76)=4.67, p<.01)$.

Two interesting aspects of the results require further discussion. First, whereas additional feedback consistently had positive effects, trimodal feedback showed no general advantage over bimodal. The touchpad with combined visual, auditory and tactile feedback did not prove to be the best solution. On the basis of the data available, it is difficult to extrapolate the meaning of this finding for real driving scenarios. It seems very likely that, once a driver listens to loud music or drives on bumpy roads, the disadvantages of one non-visual modality may be compensated for by the advantages of the other. However, this explanation needs experimental tests with real-life driving situations. Second, regarding the bimodal touchpad variants, additional auditory feedback led to somewhat shorter gazes at the display and lower workload ratings than additional tactile feedback. One reason may be that people are more used to integrating auditory feedback into their action strategies than abstract tactile feedback. More practice with Tactons may be required to achieve equal results.

\section{Conclusions}

The present study has shown that a touchpad can be a reasonable alternative to a conventional rotary push-button switch as a central input device for on-board systems in vehicles. But it also became clear that a touchpad which provides only visual feedback will not be usable while driving. Additional auditory or tactile feedback was necessary to reduce visual distraction, decrease task completion times and reduce the workload associated with the input task. In this regard, the experiment also confirms the expected advantage of multimodal over unimodal feedback. Although auditory feedback yielded somewhat better results than tactile feedback, Tactons proved to 
provide a substantial enhancement compared to unimodal visual feedback. In order to give a more detailed answer to the question of the suitability of a touchpad to control in-vehicle applications, a comparison of the multimodally enhanced touchpad with a rotary push-button with multimodal properties, such as BMW's force feedback iDrive controller, would be an interesting next step for future research.

Acknowledgments. We would like to thank Thomas Hempel and Dave Lewis for helpful comments on an earlier draft of this document.

\section{References}

1. Norman, D.A.: Psychology of Everyday Things. Basic Books, New York (1988)

2. Gaver, W.A.: Auditory Interfaces. In: Helander, M.G., Landauer, T.K., Prabhu, P.V. (eds.) Handbook of Human-Computer Interaction, pp. 1003-1041. Elsevier, Amsterdam (1997)

3. Wickens, C.D., Hollands, J.G.: Engineering Psychology and Human Performance. Prentice Hall, Upper Saddle River (2000)

4. Wickens, C.D., Sandry, D., Vidulich, M.: Compatibility and Resource Competition between Modalities of Input, Central Processing, and Output. Human Factors 25, 227-248 (1983)

5. Liu, Y.C.: Comparative Study of the Effects of Auditory, Visual and Multimodality Displays on Driver's Performance in Advanced Traveller Information Systems. Ergonomics 44, 425-442 (2001)

6. Cockburn, A., Brewster, S.A.: Multimodal Feedback for the Acquisition of Small Targets. Ergonomics 48, 1129-1150 (2005)

7. Vitense, H.S., Jacko, J.A., Emery, V.K.: Multimodal Feedback: An Assessment of Performance and Mental Workload. Ergonomics 46, 68-87 (2003)

8. Williams, J., Michelitsch, G., van Veen, J.-H., Goronzy, S.: MIAMM: Lessons Learned in Haptic \& Tactile Interaction. In: Buss, M., Fritschi, M. (eds.) Proceedings of Eurohaptics 2004, pp. 30-35. Technische Universität München, München (2004)

9. Brown, L.M., Brewster, S.A., Purchase, H.C.: A First Investigation into the Effectiveness of Tactons. In: Proceedings of Worldhaptics, pp. 167-176. IEEE, Pisa, Italy (2005)

10. Brewster, S.A., Brown, L.M.: Non-Visual Information Display Using Tactons. In: Extended Abstracts of ACM CHI 2004, pp. 787-788. ACM Press, New York (2004)

11. Mattes, S.: The Lane-Change-Task as a Tool for Driver Distraction Evaluation. In: Strasser, H., Kluth, K., Rausch, H., Bubb, H. (eds.) Quality of Work and Products in Enterprises of the Future, pp. 57-60. Ergonomia Verlag, Stuttgart (2003)

12. Byers, J.C., Bittner, A.C., Hill, S.G.: Traditional and Raw Task Load Index (TLX) Correlations: Are Paired Comparisons Necessary? In: Mital, A. (ed.) Advances in Industrial Ergonomics and Safety, pp. 481-485. I. Taylor \& Francis, London (1989)

13. Diederich, A., Colonius, H.: Bimodal and Trimodal Multisensory Enhancement: Effects of Stimulus Onset and Intensity on Reaction Time. Perception \& Psychophysics 66, 1388 1404 (2004) 